\title{
EFECTO DE BRASINOESTEROIDES EN HÍBRIDOS DE MAÍZ ANDROESTÉRILES Y FÉRTILES ${ }^{1}$
}

\author{
Baltazar Leovigildo Torres-Ruiz², Alejandro Espinosa-Calderón ${ }^{3}$, Moisés Mendoza-Rodríguez, \\ José Luis Rodríguez-de la $\mathrm{O}^{4}$, Martha Blanca Irizar-Garza ${ }^{3}$, Jaime Sahagún Castellanos-Ruiz ${ }^{4}$
}

\section{RESUMEN}

Efecto de brasinoesteroides en híbridos de maíz androestériles y fértiles. El objetivo fue determinar la respuesta de híbridos androestériles y fértiles de maíz ante la aplicación de brasinoesteroide (CIDEF-4). Se evaluaron en el Campo Experimental Valle de México, en el ciclo primavera - verano 2004 , dos dosis (30 y $60 \mathrm{~g} / \mathrm{ha}$ ) de fitohormona con su respectivo testigo en 10 genotipos, bajo un diseño de bloques completos al azar. Para la variable rendimiento no hubo diferencia significativa al aplicar CIDEF-4 en los genotipos, en el análisis general donde se engloban los 10 genotipos, sin considerar el tipo de cruzas. Mientras que al efectuar un análisis separando tipos de cruzas, se observó que al aplicar CIDEF-4 en híbridos trilineales fértiles, se tuvieron diferencias altamente significativas, donde la dosis de $30 \mathrm{~g} /$ ha rindió $8.083 \mathrm{t} / \mathrm{ha}$, respecto al testigo cuyo valor fue $3.858 \mathrm{t} / \mathrm{ha}$. En cambio en los híbridos trilineales androestériles no se presentaron diferencias significativas para las variables evaluadas. Sin embargo, en los híbridos de cruza simple en versión fértil y androestéril el brasinoesteroide adelantó la floración masculina y femenina.

Palabras clave: Zea mays L., híbridos, regulador del crecimiento, andoesterilidad, fertilidad normal.

\begin{abstract}
Brassinosteroids effect on andro-sterility and male fertiles maize hybrids. A study was conducted to evaluate the effect of brassinosteroids (CIDEF 4) on maize hybrids with male sterility and normal fertility. Ten genotypes and two doses of brassinosteroids were evaluated (30 y $60 \mathrm{~g} / \mathrm{ha}$ ), a control treatment was included without brassinosteroid application. A randomized block design with four replications was used. There was not significant difference in yield when brassinosteroid CIDEF-4 was applied to the ten genotypes. However, when the ten genotypes were classified according to hybrids types, the three-line hybrids with male fertility and brassinosteroid (CIDEF-4) were statistically different. The yield with $30 \mathrm{~g} / \mathrm{ha}$ of CIDEF-4 was 8,083 t/ha, while the check without brassinosteroid yielded 3,858 t/ha. The three-way androsterile hybrids did not show significant statistical differences for the evaluated variables. In contrast, in the single-cross hybrids with male sterility and normal fertility, the application of brassinosteroids caused earlier male and female flowering.
\end{abstract}

Key words: Zea mays L., hybrids, growth regulator, andro-sterility, normal fertility.

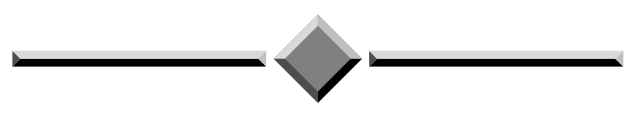

\section{INTRODUCCIÓN}

El maíz es el cultivo básico más importante en la dieta de los mexicanos, ya que el consumo anual aparente es de 209,8 kg per cápita (Morris y López 2000). En México se siembran cada año alrededor de 8,5 millones de hectáreas de maíz, con un rendimiento promedio de $2,4 \mathrm{t} / \mathrm{ha}$; la producción nacional por año

\footnotetext{
1 Recibido: 13 de noviembre, 2006. Aceptado: 29 de agosto, 2007. Trabajo de tesis de licenciatura de Ingeniero Agrónomo Fitotecnista del primer autor.

2 Departamento de Fitotecnia, Universidad Autónoma Chapingo. Correo electrónico: tr182@hotmail.com o diquiyuu@yahoo.com.mx. CP 56230.

3 Producción de Semillas, Campo Experimental Valle de México, Instituto Nacional de Investigaciones Forestales, Agrícolas y Pecuarias. México. CP.56230. Correo electrónico: espinosa.alejandro@inifap.gob.mx

4 Departamento de Fitotecnia, Universidad Autónoma Chapingo. Texcoco, Estado de México. CP 56230. Correos electrónicos: mendozaromoi@ yahoo.com.mx,jrguez@correo.chapingo.mx, martha_irizar@yahoo.com, jsahagun@correo.chapingo y mx; respectivamente.
} 
es de 16 a 18 millones de toneladas, esta producción no es suficiente para alimentar a la población en el país, por lo que se tiene que importar anualmente de seis a ocho millones de toneladas de grano para el consumo humano y animal, lo anterior es grave ya que señala que se recurre a la importación de más del $30 \%$ de grano de maíz para satisfacer las necesidades de este cereal (Sierra et al. 2004; Espinosa et al. 2007).

Mas de tres millones de productores dependen del maíz, ésto motiva a estudiar los diferentes factores que pudiesen estar relacionados, y faciliten el incremento de su productividad en México, lo que conduce a una constante formación y evaluación de híbridos de maíz, para seleccionar los mejores materiales que presenten un alto potencial en rendimiento y otras características agronómicas sobresalientes. Otra opción para elevar los rendimientos, la producción y productividad en maíz, es utilizar cada vez mejores variedades, las cuales, a partir de cierto potencial de rendimiento, podrían lograr una mejor expresión fenotípica y rendimiento, con cierto manejo agronómico, como es fertilización, densidad de población y aplicación de algunas fitohormonas (Espinosa et al. 2003).

La falta de conocimiento sobre fitohormonas y el efecto que tienen sobre la producción, ha sido una limitante en su uso, además de otros factores como el precio y los costos de aplicación. El Centro de Investigación de los Recursos Naturales de Sonora (CIDESON 1986), señala que a principios de la década de los 60`s, el Departamento de Agricultura de los Estados Unidos (USDA) dio a conocer una sustancia aislada del polen del nabo (Brassica napus L.), llamada brasinólida, que podía aumentar la producción de los cultivos agrícolas hasta un $40 \%$ con solo aplicar cantidades pequeñas como un gramo por hectárea. Como resultado se cuenta con un análogo de brasinoesteroide denominada CIDEF-4, la cual ha dado buenos resultados aumentando el rendimiento en frijol, trigo, algodón, soya, garbanzo, sorgo, avena, vid, papa, sandía, melón, brócoli, chile, zanahoria, espárrago, fresa, aguacate, tomate, pepino, mango, entre otros cultivos (CIDESON 1986).

En la actualidad la temática de los brasinoesteroides concentra la atención y esfuerzos de numerosos científicos empeñados en el aislamiento, identificación, estudio de la actividad biológica y obtención por vía sintética de nuevos integrantes de esta familia. Por otra parte, los brasinoesteroides también pueden ser considerados como un nuevo grupo de hormonas vegetales con función reguladora en el alargamiento y la división celular, a la vez interactúan con las hormonas vegetales y otras sustancias del crecimiento, todo lo que indica que los brasinoesteroides pueden jugar un papel importante en el crecimiento y desarrollo de las plantas (Terry et al. 2001).

Los brasinoesteroides son compuestos de estructura esteroidal que se caracterizan por producir la estimulación del crecimiento vegetal, el aumento de rendimiento, la producción de biomasa en diferentes cultivos y el aceleramiento de la maduración de la cosecha, además de aumentar la resistencia de las plantas a plagas y a diferentes factores de estrés como salinidad, sequía, bajas y altas temperaturas, agentes químicos agresivos como plaguicidas y herbicidas.

Las respuestas a los brasinoesteroides incluyen efectos sobre elongación, división celular, desarrollo vascular y reproductivo, la polarización de la membrana y el bombeo de protones, las relaciones fuente/ sitio de consumo y la modulación del estrés. Además interactúan con señales ambientales y pueden afectar el desarrollo de insectos y hongos, se ha evidenciado que estos compuestos incrementan la actividad de determinados metabolitos y enzimas asociadas con los mecanismos de resistencia a patógenos (Núñez y Mazorra 2001).

En maíz la aplicación del análogo de brasinoesteroide, 24-epibrasinólida, incrementó el rendimiento del cultivo, obteniéndose los mejores resultados cuando se asperjó el producto previo a la emergencia de la inflorescencia masculina. Este incremento en el rendimiento parece atribuirse al peso de 1.000 granos y el número de granos por mazorca. Los incrementos en rendimiento en la mayoría de las pruebas variaron entre 10 y $20 \%$ cuando se realizó una sola aspersión con solución de $0,01 \mathrm{ppm}$, a razón de 800 1/ha, o sea aproximadamente $8 \mathrm{mg} / \mathrm{ha}$ (Ikekawa y Zhao, citado por Núñez 1999; Fujioka y Sakurai 1993). Grajales y Hernández (2005), reportan que la aplicación de brasinoesteroides incrementó el desarrollo vegetativo de la planta del maíz híbrido Puma-1181, al aumentar la longitud de la hoja, la altura de planta y, por ende, el peso seco del tallo. 
En esta investigación se probó la respuesta de híbridos androestériles y fértiles de maíz, a la aplicación de CIDEF-4 (brasinoesteroide), con el fin de promover mejores rendimientos de grano.

\section{MATERIALES Y MÉTODOS}

El experimento se estableció en el ciclo primavera - verano del 2004, en el Campo Experimental Santa Lucía de Prías, Municipio de Texcoco, Estado de México perteneciente al Campo Experimental del Valle de México (CEVAMEX) del Instituto Nacional de Investigaciones Forestales, Agrícolas y Pecuarias (INIFAP). Se encuentra dentro del área de influencia de Chapingo, el cual de acuerdo con la clasificación climática de Köppen modificada por García (1981), tiene un clima $\mathrm{C}(\mathrm{Wo})(\mathrm{w}) \mathrm{b}\left(\mathrm{i}^{\prime}\right) \mathrm{g}$; o clima templado con lluvias en verano, el más seco de los subhúmedos, con veranos frescos y prolongados, con temperaturas, medias anuales entre 12 y $18{ }^{\circ} \mathrm{C}$; la oscilación anual de las temperaturas medias mensuales es de 5 a $7{ }^{\circ} \mathrm{C}$.

Se evaluaron 10 híbridos de maíz: tres de ellos trilineales fértiles (242E x 246) x E645, (242E x 246) x 240, (242E x 246) x 445; cuatro trilineales androestériles (244AE x 239) x E645, (241AE x 349) x IA91, (TI99AI x 27) x 106, (TI99AI x 27) x 108; dos cruzas simples fértiles ( 242 x 246), (242E x 239) x 239 y una cruza simple androestéril (242AE x 246) x 246, proporcionados por el Programa de Producción de Semillas de Maíz del CEVAMEX (Cuadro 1); y dos dosis de brasinoesteroides (30 y $60 \mathrm{~g} / \mathrm{ha}$ ) con su respectivo testigo en el cual no se aplicó la fitohormona.

La siembra se efectuó el 7 de junio del 2004 en parcelas de $5 \mathrm{~m}$ de longitud, con una distancia entre surcos de $80 \mathrm{~cm}$, depositando tres semillas por golpe a 0,50 m cada uno. Se empleó un surco por parcela.

Los 30 tratamientos se distribuyeron en un diseño experimental de bloques completos al azar con cuatro repeticiones. Se realizaron tres aplicaciones, en el primer tratamiento la dosis asperjada fue a razón de $10 \mathrm{~g} / \mathrm{h}$ a por lo que la aplicación final fue de $30 \mathrm{~g} / \mathrm{ha}$, en la otra dosis de brasinoesteroides, se realizaron tres aplicaciones con dosis asperjada de $20 \mathrm{~g} / \mathrm{ha}$, en
Cuadro 1. Híbridos de maíz trilineales y de cruza simple androestériles y fértiles, utilizados en aplicaciones de diferentes dosis de brasinoesteroides (CIDEF4), para evaluar el efecto en rendimiento. Campo Experimental del Valle de México (CEVAMEX), Instituto Nacional de Investigaciones Forestales, Agrícolas y Pecuarias (INIFAP), Texcoco, México. Ciclo primavera - verano, 2004.

\begin{tabular}{|c|c|c|}
\hline Genotipo & Tipo de híbrido & $\begin{array}{c}\text { Androesteril/ } \\
\text { Fértil }\end{array}$ \\
\hline$(242 E x 246) \times$ E645 & Trilineal & Fértil \\
\hline$(242 \mathrm{Ex} 246) \times 240$ & Trilineal & Fértil \\
\hline$(242 \operatorname{Ex} 246) \times 445$ & Trilineal & Fértil \\
\hline$(244 \mathrm{AEx} 239) \times \mathrm{E} 645$ & Trilineal & Androesteril \\
\hline (241AEx349) x IA91 & Trilineal & Androesteril \\
\hline (TI99A15x27) x 106 & Trilineal & Androesteril \\
\hline (TI99A15x27) x 108 & Trilineal & Androesteril \\
\hline$(242 \mathrm{AEx} 246) \times 246$ & Simple & Androesteril \\
\hline$(242 E \times 239) \times 239$ & Simple & Fértil \\
\hline $242 \times 246$ & Simple & Fértil \\
\hline
\end{tabular}

cada aplicación, por lo cual la final fue de $60 \mathrm{~g} / \mathrm{ha}$. La dilución en agua fue a razón de 200 litros de agua por hectárea. Las aspersiones se llevaron a cabo el 6, 13 y 20 de julio, es decir a los 29, 36 y 43 días después de la siembra.

El cultivo se desarrolló con dos riegos de auxilio, y posteriormente, bajo condiciones de temporal, manejándose la fórmula de fertilización 160 - 70 - 30, en dos aplicaciones, la mitad del nitrógeno, todo el fósforo y todo el potasio en la siembra, la otra mitad del nitrógeno en la segunda escarda. Las variables evaluadas fueron: longitud de mazorca (LM), hileras por mazorca $(\mathrm{NH})$, granos por hilera $(\mathrm{GH})$, peso de 50 granos (P50G), porcentaje de humedad del grano (PORH), peso hectolítrico $(\mathrm{PH})$, tamaño de grano (TG) y rendimiento $(\mathrm{R})$. El análisis estadístico se realizó como factorial, considerando los 10 híbridos, así como las dosis de brasinoesteroides, en forma paralela, con base en el tipo de conformación del genotipo y su condición fértil o androestéril. Se realizaron análisis estadísticos, agrupando por separado híbridos fértiles, así como androestériles, en todos los casos se utilizó comparación de medias de Tukey. 


\section{RESULTADOS Y DISCUSIÓN}

En los resultados obtenidos en el factor genotipos se presentaron diferencias significativas para rendimiento $(\leq 0,01)$ y para número de hileras $(\leq 0,05)$. Para el factor de variación dosis sólo se detectó significancia para granos por hilera $(\leq 0,05)$. En el factor de variación correspondiente a la interacción genotipos $\mathrm{x}$ dosis se presentaron diferencias estadísticas altamente significativas para las variables rendimiento, granos por hilera y longitud de mazorca $(\leq 0,01)$ y significativas $(\leq 0,05)$, para número de hileras (Cuadro 2$)$.

Los genotipos fértiles fueron en su mayoría los que tuvieron una mayor expresión en la variable rendimiento y por consiguiente, en el resto de las variables (Cuadro 3), esto es debido probablemente al origen de cada genotipo, lo cual se puede contrastar con los genotipos androestériles. Cabe aclarar que los genotipos androestériles si bien fueron polinizados por otros genotipos fértiles ya que todo el experimento se rodeo con materiales de fertilidad normal, para que no hubiese problemas de falta de fecundación, este factor debe ser asegurado en siguientes trabajos.

Se considera que los rendimientos de grano fueron bajos, con respecto al potencial de rendimiento de los híbridos empleados, y su expresión en fechas de siembra tempranas. Esto se debió en primer término, a la fecha de siembra retrasada, es decir en junio, además de las condiciones del ambiente ya que los híbridos responden bien a ambientes favorables con buena

Cuadro 2. Comparación de medias para rendimiento ( $\mathrm{R})$, número de hileras $(\mathrm{NH})$, granos por hilera $(\mathrm{GH})$ y longitud de mazorca (LM), en 10 genotipos de maíz y tres dosis de CIDEF-4, Campo Experimental del Valle de México (CEVAMEX), Instituto Nacional de Investigaciones Forestales, Agrícolas y Pecuarias (INIFAP)Texcoco, México. Ciclo primavera - verano, 2004.

\begin{tabular}{|c|c|c|c|c|}
\hline \multirow{2}{*}{$\begin{array}{c}\text { Factores } \\
\text { Genotipos }\end{array}$} & \multicolumn{4}{|c|}{ Variables estudiadas } \\
\hline & $\begin{array}{c}\mathbf{R} \\
(\mathbf{t} / \mathbf{h a})\end{array}$ & NH & GH & $\begin{array}{l}\mathbf{L M} \\
(\mathrm{cm})\end{array}$ \\
\hline$(242 E \times 246) \times E^{2} 645^{1}$ & $6.191 \mathrm{a}$ & 14,16 ba & $32,25 \mathrm{a}$ & $14.300 \mathrm{ba}$ \\
\hline$\left(\right.$ TI99A15x27) x $108^{2}$ & $6.191 \mathrm{a}$ & $14,00 \mathrm{ba}$ & $32,33 \mathrm{a}$ & 14.966 ba \\
\hline$(242 \operatorname{Ex} 246) \times 240^{1}$ & $6.150 \mathrm{a}$ & 14,16 ba & 29,66 a & $14.225 \mathrm{ba}$ \\
\hline $242 \times 246^{4}$ & $6.016 \mathrm{a}$ & $14,50 \mathrm{ba}$ & $32,25 \mathrm{a}$ & $15.750 \mathrm{a}$ \\
\hline$(\mathrm{TI} 99 \mathrm{~A} 15 \times 27) \times 106^{2}$ & $5.891 \mathrm{a}$ & $15,33 \mathrm{a}$ & 29,41 a & $13.308 \mathrm{~b}$ \\
\hline$(241 \mathrm{AEx} 349) \times$ IA91 ${ }^{2}$ & 5.316 ba & $15,08 \mathrm{ba}$ & 29,66 a & $13.608 \mathrm{ba}$ \\
\hline$(242 \operatorname{Ex} 239) \times 239^{4}$ & $5.100 \mathrm{bac}$ & 14,41 ba & $30,50 \mathrm{a}$ & $13.991 \mathrm{ba}$ \\
\hline$(242 \operatorname{Ex} 246) \times 445^{1}$ & $4.350 \mathrm{bc}$ & $13,91 \mathrm{~b}$ & $29,58 \mathrm{a}$ & $13.458 \mathrm{~b}$ \\
\hline$(242 \mathrm{AEx} 246) \times 246^{3}$ & $4.225 \mathrm{bc}$ & 14,66 ba & $30,00 \mathrm{a}$ & $13.508 \mathrm{~b}$ \\
\hline$(244 \mathrm{AEx} 239) \times \mathrm{E} 645^{2}$ & $3.716 \mathrm{c}$ & $13,91 \mathrm{~b}$ & $28,08 \mathrm{a}$ & $13.294 \mathrm{~b}$ \\
\hline D.S.H. $(0,05)$ & 1.527 & 1,37 & 4,64 & 2.152 \\
\hline \multicolumn{5}{|l|}{ Dosis (g/ha) } \\
\hline 0 & $5.232 \mathrm{a}$ & $14.375 \mathrm{a}$ & 30.775 ba & $14.417 \mathrm{a}$ \\
\hline 30 & $5.410 \mathrm{a}$ & $14.675 \mathrm{a}$ & $29.025 \mathrm{~b}$ & $13.440 \mathrm{~b}$ \\
\hline 60 & $5.302 \mathrm{a}$ & $14.200 \mathrm{a}$ & $31.325 \mathrm{a}$ & $14.265 \mathrm{ba}$ \\
\hline D.S.H. $(0,05)$ & 0,614 & 0,552 & 1.867 & 0,865 \\
\hline
\end{tabular}

$1=$ trilineal fértil; $2=$ trilineal androesteril; $3=$ simple androesteril; $4=$ simple fértil; medias con la misma letra no presenta diferencias significativas, de acuerdo a la prueba de Tukey, con $\alpha=0,05$. 
Cuadro 3. Comparación de medias para rendimiento ( $\mathrm{R})$, número de hileras $(\mathrm{NH})$, granos por hilera $(\mathrm{GH})$ y longitud de mazorca $(\mathrm{LM})$, para tres híbridos trilineales fértiles de maíz y tres dosis de CIDEF-4, Campo Experimental del Valle de México (CEVAMEX), Instituto Nacional de Investigaciones Forestales, Agrícolas y Pecuarias (INIFAP), Texcoco, México. Ciclo primavera - verano, 2004.

\begin{tabular}{lllll}
\hline \multicolumn{1}{c}{ Factores } & \multicolumn{5}{c}{ Variables estudiadas } \\
\hline \multicolumn{1}{c}{ Genotipos } & $\begin{array}{c}\text { R } \\
(\mathbf{t} / \mathbf{h a})\end{array}$ & NH & $\mathbf{G H}$ & $\begin{array}{c}\text { LM } \\
(\mathbf{c m})\end{array}$ \\
\hline$(242 \mathrm{Ex} 246) \times \mathrm{E} 645$ & $6.191 \mathrm{a}$ & $14.166 \mathrm{a}$ & $32.250 \mathrm{a}$ & $14.300 \mathrm{a}$ \\
$(242 \mathrm{Ex} 246) \times 240$ & $5.933 \mathrm{ba}$ & $15.000 \mathrm{a}$ & $29.833 \mathrm{a}$ & $13.991 \mathrm{a}$ \\
$(242 \mathrm{Ex} 246) \times 445$ & $5.100 \mathrm{~b}$ & $14.416 \mathrm{a}$ & $30.500 \mathrm{a}$ & $13.583 \mathrm{a}$ \\
Dosis (g/ha) & & & & \\
0 & $3.858 \mathrm{c}$ & $14.583 \mathrm{~b}$ & $27.667 \mathrm{~b}$ & $12.350 \mathrm{~b}$ \\
30 & $8.083 \mathrm{a}$ & $15.750 \mathrm{a}$ & $32.083 \mathrm{a}$ & $14.333 \mathrm{a}$ \\
60 & $5.283 \mathrm{~b}$ & $13.250 \mathrm{c}$ & $32.833 \mathrm{a}$ & $15.191 \mathrm{a}$ \\
DMS & 1.254 & 1.349 & 3.564 & 1.417 \\
\hline
\end{tabular}

Medias con la misma letra no presenta diferencias significativas, de acuerdo a la prueba de Tukey, con $\alpha=0,05$.

precipitación pluvial, sin limitantes de humedad. Es importante mencionar que el experimento fue conducido con el auxilio de dos riegos, aún así las condiciones de humedad no fueron suficientes ya que el resto del ciclo prevalecieron condiciones de escasa humedad, además, de la siembra retrasada con sus inconvenientes en temperaturas, es posible que estos factores tan complejos hayan influido para que los genotipos mostraran una menor expresión en el rendimiento, sin embargo se puede afirmar que en general los rendimientos alcanzados por los genotipos, fueron aceptables para aquellos materiales de menor sensibilidad.

Para el caso del factor dosis, considerando los 10 genotipos, la comparación de medias para la variable rendimiento, se confirma que no existe diferencia significativa, lo que señala que no hubo efecto al aplicar el brasinoesteroide (CIDEF-4), este tipo de respuesta ya había sido reportado en variedades, híbridos varietales e híbridos de Valles Altos (Espinosa et al. 2003), tampoco existieron diferencias en el número de hileras, pero si se presentaron diferencias en grupos estadísticos en las variables granos por hilera y longitud de mazorca, donde el testigo expresó los valores más altos (Cuadro 2). La aplicación de la fitohormona se hizo a los 29, 36 y 43 días después de la siembra es decir en la fase vegetativa de la planta, caso contrario a Ikekawa y Zhao, citado por Nuñez (1999), quien dice que la aplicación de la 24-epibrasinólida en maíz, incrementó el rendimiento del cultivo, obteniéndose los mejores resultados cuando se asperjó el producto previo a la emergencia de la inflorescencia masculina.

Con respecto a la interacción genotipos $\mathrm{x}$ dosis $(\mathrm{G}$ $\mathrm{x}$ D), en las comparaciones de medias, las dosis dentro de los genotipos, fueron diferentes estadísticamente, lo que implica que la respuesta de los genotipos fue diferencial ante la aplicación de dosis de brasinoesteroides, y como menciona Andrade et al. (1996), el uso de fitohormonas (CIDEF-4) aplicadas en el cultivo en la fase vegetativa, logra una cierta expresión fenotípica lo que puede depender del genotipo.

Después del análisis de varianza general para todos los genotipos, al no tener claro si hay respuesta del brasinoesteroide usado, por la confusión o efecto que puede propiciar el tipo de conformación del genotipo y su condición fértil o androesteril, se separaron algunos 
genotipos para hacer un análisis por grupo, con la finalidad de tener un panorama más amplio acerca del comportamiento de los genotipos por efecto de la aplicación del CIDEF-4. Esta separación se realizó de acuerdo al tipo de conformación de híbridos, teniendo como resultados tres grupos, cada uno de ellos formado por híbridos trilineales fértiles, híbridos trilineales androestériles e híbridos de cruza simple, con una retro cruza hacia un progenitor en versión fértil y androestéril.

En el caso de los híbridos trilineales fértiles, en el análisis de varianza, para rendimiento se detectaron diferencias estadísticas para genotipos y diferencias altamente significativas para el tratamiento dosis de brasinoesteroides, así como diferencias estadísticas para la interacción genotipos $\mathrm{x}$ dosis. Para el factor de variación dosis de brasinoesteroides el análisis de varianza determinó diferencias estadísticas $(0,01$ de probabilidad) para las variables número de hileras, granos por hilera y longitud de mazorca, así como diferencias estadísticas $(0,05$ de probabilidad) para granos por hilera y longitud de mazorca.

En la comparación de medias para los híbridos trilineales fértiles, el rendimiento más elevado (8.033 $\mathrm{t} / \mathrm{ha}$ ), bajo la dosis con mejor respuesta fue la de $30 \mathrm{~g} / \mathrm{ha}$ respecto al testigo que produjo $3.858 \mathrm{t} / \mathrm{ha}$, por lo que de manera implícita el resto de los componentes de rendimiento $\mathrm{NH}$, GH y LM mostraron también diferencias altamente significativas (Cuadro 3). Estos resultados concuerda con Ikekawa y Zhao (1991), quienes mencionan que la aplicación de la 24-epibrasinólida en maíz, incrementó el rendimiento del cultivo, en la mayoría de las pruebas, este incremento osciló entre 10 y 20\%. Así como el Centro de Investigación y Desarrollo de los Recursos Naturales de Sonora señala que se pueden tener incrementos en los rendimientos hasta de un 100\%. Este resultado es interesante, ya que al separarse un grupo sólo de materiales fértiles, puede ser que se separan efectos compensatorios, por la condición androesteril o fértil, que enmascaran el efecto en el análisis.

Con respecto a los híbridos trilineales androestériles, los genotipos no presentaron diferencias significativas en las variables $\mathrm{R}, \mathrm{NH}$, y LM, pero si en $\mathrm{GH}$, sin embargo, estas tres últimas forman parte del componente del rendimiento, por lo que se da énfasis a la primera variable que es de importancia agronómica. Lo mismo sucede, con el factor dosis ya que de acuerdo a la comparación de medias, al aplicar CIDEF-4, los componentes del rendimiento no mostraron diferencias significativas respecto al testigo, esto quiere decir que la androesterilidad de los híbridos podría ser algún factor que influencia que no haya respuesta ante la aplicación de brasinoesteroides (Cuadro 4). Sería interesante evaluar materiales similares, es decir isogénicos, con aplicación de fitohormonas, para esclarecer su respuesta en función de la condición de androesterilidad o fertilidad.

Con respecto a la comparación de medias, los híbridos androestériles se ubicaron en un solo grupo de significancia, en las variables $\mathrm{R}, \mathrm{NH}$, y LM, pero si en GH (Cuadro 4), es interesante señalar que dos de los híbridos evaluados tienen como progenitor a la misma hembra y la diferencia radica en el macho. En los materiales evaluados, la androesterilidad podría ser un elemento que pudo influir en la similitud estadística, ante la aplicación de brasinoesteroides, ya que al no producir polen, se podría generar fisiológicamente algún tipo de interacción, por lo que en futuros trabajos este asunto puede ser revisado con mayor detenimiento.

En el caso de los híbridos de cruza simple, con una retrocruza hacia uno de los progenitores, no se detectaron diferencias en el factor genotipos en la variable rendimiento y sus componentes, sin embargo se presentó diferencia altamente significativa para la aplicación de dosis de brasinoesteroides, lo que confirma la necesidad de separar a los genotipos por condición de fertilidad, para limitar el factor compensatorio que se podría generar en las versiones androestériles y fértiles. Las diferencias probablemente se deban a que tienen efecto de la retrocruza ya que la cruza simple usada como hembra se cruza con una línea que integra la cruza simple, es decir una línea progenitora (Cuadro 5).

La comparación de medias señala la similitud en grupos estadísticos de las tres cruzas simples, así como similitud en las otras variables evaluadas. En cambio en la comparación de las dosis aplicadas, el testigo sin aplicar, mostró el rendimiento más elevado (6.275 t/ha), similar a la aplicación de brasinoesteroide en dosis de $30 \mathrm{~g} / \mathrm{ha}$, al aplicar la dosis de $60 \mathrm{~g} / \mathrm{ha}$ el rendimiento fue menor estadísticamente (1.466 t/ha), para el mismo factor dosis, en número de hileras no hubo diferencias estadísticas, pero si en granos por hilera y longitud de 
Cuadro 4. Comparación de medias para rendimiento ( $\mathrm{R})$, número de hileras $(\mathrm{NH})$, granos por hilera $(\mathrm{GH})$ y longitud de mazorca $(\mathrm{LM})$, para híbridos trilineales androestériles de maíz y tres dosis de CIDEF-4,Campo Experimental del Valle de México (CEVAMEX), Instituto Nacional de Investigaciones Forestales, Agrícolas y Pecuarias (INIFAP), Texcoco, México. Ciclo primavera - verano, 2004.

\begin{tabular}{|c|c|c|c|c|}
\hline \multirow{2}{*}{$\begin{array}{l}\text { Factores } \\
\text { Genotipos }\end{array}$} & \multicolumn{4}{|c|}{ Variables estudiadas } \\
\hline & $\mathbf{R}$ (t/ha) & NH & GH & LM (cm) \\
\hline (TI99A15x27) x 106 & $6.133 \mathrm{a}$ & $13.833 \mathrm{a}$ & $33.000 \mathrm{a}$ & $15.050 \mathrm{a}$ \\
\hline (241AEx349) x IA91 & $5.691 \mathrm{a}$ & $14.083 \mathrm{a}$ & $28.833 \mathrm{~b}$ & $14.083 \mathrm{a}$ \\
\hline$(244 \mathrm{AEx} 239) \times \mathrm{E} 645$ & $5.675 \mathrm{a}$ & $14.083 \mathrm{a}$ & $32.167 \mathrm{ba}$ & $15.566 \mathrm{a}$ \\
\hline (TI99A15x27) x 108 & $5.200 \mathrm{a}$ & $14.166 \mathrm{a}$ & $32.250 \mathrm{ba}$ & $15.091 \mathrm{a}$ \\
\hline DMS & 1.729 & 1.003 & 3,93 & 2.235 \\
\hline \multicolumn{5}{|l|}{ Dosis (g/ha) } \\
\hline 0 & $5.906 \mathrm{a}$ & $14.375 \mathrm{a}$ & $31.875 \mathrm{a}$ & $15.525 \mathrm{a}$ \\
\hline 30 & $5.468 \mathrm{a}$ & $13.812 \mathrm{a}$ & $30.625 \mathrm{a}$ & $14.212 \mathrm{a}$ \\
\hline 60 & $5.650 \mathrm{a}$ & $13.937 \mathrm{a}$ & $32.188 \mathrm{a}$ & $15.106 \mathrm{a}$ \\
\hline DMS & 1.358 & 0.788 & 3.087 & 1.756 \\
\hline
\end{tabular}

Medias con la misma letra no presenta diferencias significativas, de acuerdo a la prueba de Tukey, con $\alpha=0,05$.

Cuadro 5. Comparación de medias para rendimiento $(\mathrm{R})$, número de hileras $(\mathrm{NH})$, granos por hilera (GH) y longitud de mazorca (LM), para híbridos de cruza simple de maíz y tres dosis de CIDEF-4, Campo Experimental del Valle de México (CEVAMEX), Instituto Nacional de Investigaciones Forestales, Agrícolas y Pecuarias (INIFAP), Texcoco, México. Ciclo primavera - verano, 2004.

\begin{tabular}{lllll}
\hline Factores & \multicolumn{4}{c}{ Variables estudiadas } \\
\hline Genotipos & R (t/ha) & NH & GH & LM $(\mathbf{c m})$ \\
\hline $242 \times 246^{1}$ & $4.716 \mathrm{a}$ & $14.416 \mathrm{a}$ & $28.500 \mathrm{a}$ & $13.008 \mathrm{a}$ \\
$(242 \mathrm{AEx} 246) \times 246^{2}$ & $4.250 \mathrm{a}$ & $15.333 \mathrm{a}$ & $29.167 \mathrm{a}$ & $13.250 \mathrm{a}$ \\
$(242 \mathrm{Ex} 239) \times 239^{1}$ & $3.866 \mathrm{a}$ & $14.666 \mathrm{a}$ & $26.833 \mathrm{a}$ & $12.233 \mathrm{a}$ \\
\hline Dosis (g/ha) & & & & \\
0 & $6.275 \mathrm{a}$ & $14.583 \mathrm{a}$ & $31.917 \mathrm{a}$ & $14.025 \mathrm{a}$ \\
30 & $5.091 \mathrm{a}$ & $15.333 \mathrm{a}$ & $29.000 \mathrm{a}$ & $13.525 \mathrm{a}$ \\
60 & $1.466 \mathrm{~b}$ & $14.500 \mathrm{a}$ & $23.583 \mathrm{~b}$ & $10.941 \mathrm{~b}$ \\
DMS & 1.335 & 1.238 & 4.222 & 1.665 \\
\hline
\end{tabular}

1= fértil; 2 = androestéril; medias con la misma letra no presenta diferencias significativas, de acuerdo a la prueba de Tukey, $\operatorname{con} \alpha=0,05$.

mazorca, donde el testigo y la dosis de $30 \mathrm{~g} / \mathrm{ha}$, propiciaron mejor expresión en comparación con la dosis de
$60 \mathrm{~g} / \mathrm{ha}$ (Cuadro 5). Esto se puede deber a lo que plantea Sasse (1997), que los efectos de los brasinoesteroides 
naturales en el campo, pueden ser de corta duración o esporádicos, por lo que debe ser útil la aplicación de compuestos de más larga duración, que sean transformados en las plantas a brasinoesteroides activos.

\section{CONCLUSIONES}

El rendimiento, no se vio influenciado por el efecto de brasinoesteroide en las dosis de 30 y $60 \mathrm{~g} / \mathrm{ha}$, al hacer el análisis conjunto para los diez genotipos. Lo cual se atribuye a la conformación genética y características fértiles y androestériles de dichos genotipos.

Un análisis por grupo, con base en el tipo de cruzas, conformación trilineal o simple y fértil o androestéril, la aplicación de CIDEF-4, si mostró efecto en el rendimiento de los genotipos trilineales fértiles (242Ex246) x E645, (242Ex246) x 240 y (242Ex246) $\mathrm{x} 445$, siendo la dosis de $30 \mathrm{~g} / \mathrm{ha}$ (8.083 t/ha) superior a la del testigo (3.858 t/ha).

El efecto del CIDEF-4 en el rendimiento para los híbridos trilineales fértiles fue diferente en comparación de los genotipos androestériles donde no se mostró dicho efecto. Sin embargo en los híbridos de cruza simple en versión fértil y androestéril el brasinoesteroide afectó negativamente el rendimiento en comparación con el testigo sin aplicación.

\section{LITERATURA CITADA}

CIDESON (Centro de Investigación de los Recursos Naturales de Sonora). 1986. Boletín. CIDEF-4, fitohormona esteroidal. Elaborada por: Fitohormonas de Sonora, S. A. de C. V. Hermosillo Sonora.

ESPINOSA, A.; MUSITO, N.; TADEO, M.; MARTÍNEZ, R. 2003. Respuesta a la aplicación de brasinoesteroides en híbridos y variedades de maíz de calidad proteínica y normal de Valles Altos. In: Memoria de VI Congreso Internacional en Ciencias Agrícolas, Universidad Autónoma de Baja California, Instituto de Ciencias Agrícolas, Mexicali, B.C. p 359-364.

ESPINOSA C., A.; TURRENT, A.; TADEO, M.; GÓMEZ, N.; SIERRA M.; PALAFOX, A.; CABALLERO, F.;
RODRÍGUEZ, F.; VALDIVIA, R.; ESQUEDA, E. 2007. Algunos elementos de la crisis del maíz y la tortilla en México. In: Resúmenes del LIII Reunión Anual del Programa Cooperativo Centroamericano para el Mejoramiento de Cultivos y Animales (PCCMCA). Instituto de Ciencias y Tecnología Agropecuaria, ICTA, Guatemala. p. 93-93.

FUJIOKA, S.; SAKURAI, A. 1993. The current of physiology and biochemistry of brassinosteroids. Plant Growth Regulation 13(2): 147 - 159.

GRAJALES, O.; HERNÁNDEZ, M. 2005. Los brasinoesteroides y el desarrollo del maíz híbrido Puma 1181. Revista FESC Divulgación Científica y Multidisciplinaria. Facultad de Estudios Superiores Cuautitlán, México D. F. 4 (17): 5-7.

MORRIS, M. L.; LÓPEZ, M.A. 2000. Impactos del mejoramiento de maíz en América Latina 1966-1997. México, D.C. México. Centro Internacional de Mejoramiento de Maíz y Trigo. 45 p.

NÚÑEZ, M. 1999. Reseña bibliográfica: Aplicaciones prácticas de los brasinoesteroides y sus análogos en la agricultura. Cultivos Tropicales (Cuba) 20 (3):63-72.

; Mazorra M., L. 2001. Revisión bibliográfica: Los brasinoesteroides y la respuesta de las plantas al estrés. Cultivos Tropicales (Cuba) 22 (3):19-26.

SASSE, M. 1997. Recent progress in brassinosteroid research. Physiologia Plantarum 100(3): 696-701.

SIERRA, M.; MÁRQUEZ, F.; VALDIVIA, R.; CÓRDOVA, H.; LEZAMA, R.; PESCADOR, A. 2004. Uso de probadores en la selección de líneas para formar híbridos de maíz (Zea mays L.). Agricultura Técnica en México. Vol.30 Núm. 2, Julio - Diciembre 2004. 169-181.

TERRY, E.; NÚÑEZ, M.; PINO, M.; MEDINA, N. 2001. Efectividad de la combinación biofertilizantes - análogo de brasinoesteroides en la nutrición del tomate (Licopersicon esculentum Mill). Cultivos Tropicales 20 (2): 59-65. 